\title{
The charged bouncing ball: An experimental model for period-doubling bifurcation
}

\author{
C. Soria, A. Ramos and A. T. Pérez \\ Departamento de Electrónica y Electromagnetismo, Facultad de Física \\ Avda. Reina Mercedes s/n. 41012 Sevilla, Spain
}

(received 30 October 1996; accepted 6 February 1997)

PACS. 47.65+a - Magnetohydrodynamics and electrohydrodynamics.

PACS. $47.52+\mathrm{j}-$ Chaos.

PACS. 41.20Cv - Electrostatics; Poisson and Laplace equations, boundary-value problems.

\begin{abstract}
This paper presents an experimental and theoretical study of the dynamics of a conducting ball in a poorly conducting liquid subjected to an electric field. When the applied voltage is constant the ball bounces regularly on the lower electrode. If an $\mathrm{AC}$ voltage is superimposed, with a period equal to the unperturbed time between impacts, the ball undergoes a period-doubling bifurcation when increasing the amplitude of the AC signal. The non-linear map which describes the dynamics of the ball is closely related to the standard map and to the classical problem of a bouncing ball on a moving table.
\end{abstract}

Introduction. - The problem of a bouncing ball on a moving table is a classical problem of dynamics. It has played an important role as a model for non-linear systems [1]. Also it has been of interest since Fermi proposed a simplified model for the acceleration of cosmic rays by random oscillating magnetic fields [2].

The system we study was already considered by Haus and Melcher [3], as a system which clearly demonstrated the effect of charge relaxation time. A conducting sphere is made to bounce between parallel plate electrodes immersed in a slightly conducting fluid. The application of high voltage charges the sphere when being in contact with the lower electrode. Above a certain voltage, at which the Coulomb force balances the weight, the sphere rises, but its charge leaks away, due to the finite charge relaxation time, and it returns to the electrode. The time of flight is a non-linear function of the applied voltage.

When applying a fixed DC voltage the sphere bounces regularly, with a very well-defined time between impacts. When an AC voltage is superimposed, the dynamics becomes more complex and there appears all the phenomenology associated to bifurcations in non-linear systems. In particular, when the frequency of the AC field is close to the natural frequency of bouncing there is a period-doubling bifurcation: that is, for a certain value of the AC amplitude, the sphere starts to bounce alternatively with two different times between impacts.

Theoretical model. - The equation of motion of a ball of radius $R$ and density $\rho_{\mathrm{s}}$ immersed in a liquid of density $\rho_{\mathrm{l}}$ is

$$
m_{\mathrm{e}} \frac{\mathrm{d} v}{\mathrm{~d} t}=F_{\mathrm{e}}+F_{\mathrm{g}}+F_{\mathrm{d}},
$$

where $m_{\mathrm{e}}=\left(\rho_{\mathrm{s}}+0.5 \rho_{\mathrm{l}}\right) 4 \pi R^{3} / 3$ is an effective ball mass [4], including the inertia of the liquid around the ball, $v$ is the velocity in vertical direction and $F_{\mathrm{e}}, F_{\mathrm{g}}$ and $F_{\mathrm{d}}$ are, respectively, the electric force, the gravity force and the drag. Let us consider each force separately.

(C) Les Editions de Physique 


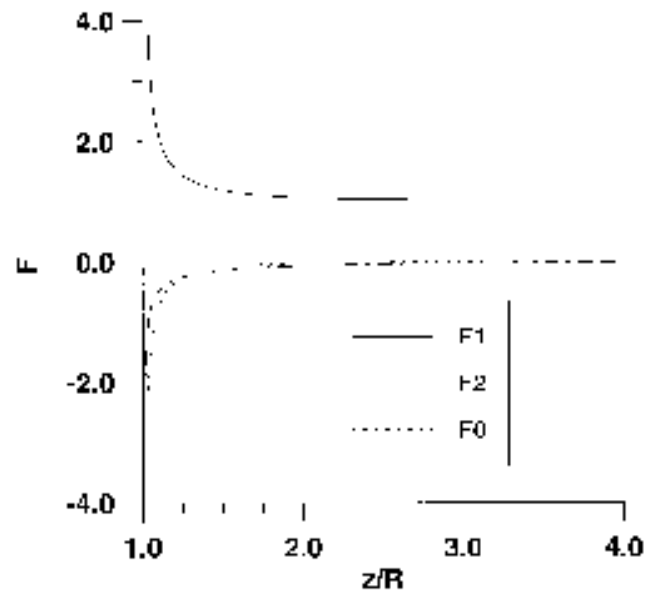

Fig. 1. - The three functions involved in the calculation of the electric force.

The electric force on a conducting particle exerted by an external electric field in the presence of an electrode was treated by Felici [5], who showed how to compute the force in the general case. He considered two cases in detail, that of a particle at the same potential as the electrode, and that of a particle that after the contact with the electrode remains isolated. In our case we need the general expression of the electric force on a sphere as a function of the distance to the electrode for any given charge. This force can be expressed

$$
F_{\mathrm{e}}=4 \pi \epsilon_{1} R^{2} E_{0}^{2} F_{0}(z / R)+q E_{0} F_{1}(z / R)+\frac{q^{2}}{4 \pi \epsilon_{l} R^{2}} F_{2}(z / R)
$$

where $\epsilon_{\mathrm{l}}$ is the liquid permittivity, $R$ the radius of the sphere, $E_{0}$ the external electric field, $q$ the charge of the ball and $z$ the distance from the center of the sphere to the electrode. $F_{0}, F_{1}$ and $F_{2}$ are non-dimensional functions of $z / R$ only. The first term represents a dipole-dipole interaction, the second one the force due to the imposed electric field, and the third one is the force between the charge and its image. The three functions are very well approximated for $z / R>4$ by

$$
F_{0}=-\frac{3 R^{4}}{8 z^{4}}, \quad F_{1}=1+\frac{R^{3}}{2 z^{3}}, \quad F_{2}=-\frac{R^{2}}{4 z^{2}} ;
$$

the deviation from this behavior for $z / R<4$ is due to the finite size of the sphere, that requires a distribution of dipole and charge to solve the problem. Figure 1 shows the three functions for $z / R<4$.

In contact with the electrode the ball takes a charge [5] $q_{0}=4 \pi \epsilon_{1} R^{2}\left(\pi^{2} / 6\right) E_{0}$. This charge leaks away as the ball rises due to the conductivity of the liquid. At any time $t$ after the impact the charge is

$$
q(t)=q_{0} e^{-t / \tau}
$$

where $\tau=\epsilon_{l} / \sigma$ ( $\sigma$, liquid conductivity) is the electric relaxation time.

The gravity force is the weight of the ball $m g$ minus the buoyancy $m_{1} g$, where $m_{1}$ is the mass of the corresponding volume of liquid. Finally the drag force is due to the viscosity $\nu$ of the liquid. The expression of this force depends on the Reynolds number associated with the ball motion $R e=v R / \nu$. In our experiments (see below) for a typical jump is $v \sim 0.01 \mathrm{~m} / \mathrm{s}$ and $R e \sim 0.6<1$. Therefore the Reynolds number is low enough for the Stokes law to apply and 


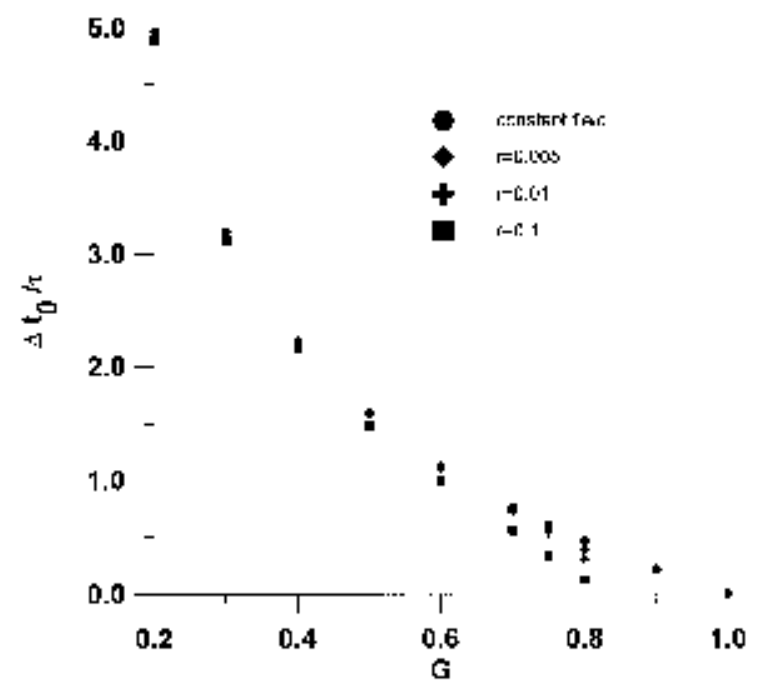

Fig. 2. - Non-dimensional time of flight vs. the parameter $G=\left(E_{\mathrm{c}} / E_{0}\right)^{2}$. The parameter $r$ is defined in the text.

we can write $F_{\mathrm{d}}=6 \pi \rho_{\mathrm{l}} \nu R v$. A further simplification can be made if we estimate the time of acceleration. A comparison of the inertial term $m_{\mathrm{e}} \mathrm{d} v / \mathrm{d} t$ with the drag force in our experiments provides a time scale for the inertial term: $t_{\mathrm{i}} \sim m_{\mathrm{e}} /\left(6 \pi \rho_{\mathrm{l}} \nu R\right) \sim 0.04 \mathrm{~s}$. As a typical jump takes 1 to 2 seconds, the inertial term is negligible and the equation of motion (1) reduces to

$$
6 \pi \rho_{\mathrm{l}} \nu R v=F_{\mathrm{e}}-\left(m-m_{\mathrm{l}}\right) g .
$$

As a first approximation consider $F_{\mathrm{e}}=q(t) E_{0}=q_{0} E_{0} e^{-t / \tau}$. Then the equation is readily integrated and from it a relation between the time of flight $\Delta t_{0}$ and the applied field is obtained:

$$
1-e^{-\Delta t_{0} / \tau}=\frac{\left(m-m_{1}\right) g}{q_{0} E_{0}} \frac{\Delta t_{0}}{\tau} .
$$

The non-dimensional parameter $G=\left(\left(m-m_{1}\right) g\right) /\left(q_{0} E_{0}\right)=\left(E_{\mathrm{c}}^{2}\right) /\left(E_{0}^{2}\right)$ represents the ratio between the gravity force and the electric force. $G$ has to be less than one in order to have motion, and therefore $E_{\mathrm{c}}=\sqrt{3\left(m-m_{1}\right) g /\left(2 \pi^{3} \epsilon_{1} R^{2}\right)}$ represents the minimum field needed to lift the ball. Figure 2 shows the time of flight vs. $G$ calculated from eq. (6).

We have also solved eq. (5) numerically using the general form of the electric-force equation (2). Another non-dimensional parameter appears in this case, $r=6 \pi \rho \nu R^{2} /\left(m-m_{1}\right) g \tau$, related to the fact that the force depends on the radius of the sphere. Figure 2 shows the results for the time of flight vs. $G$ for three different values of $r$. In our experiments $r$ is of order 0.005 and it is clear from the figure that the case of a constant field is a very good approximation. However, the minimum field needed to lift the ball is increased if eq. (2) is considered: when the ball is in contact with the electrode $F_{\mathrm{e}}=0.832 q_{0} E_{0}$ instead of $q_{0} E_{0}$. In any case, if the ball jumps are longer than 2 or 3 ball radii, the electric force can be taken as $q E_{0}$ without any significant error and we are going to use this approximation in the rest of the paper.

If an AC voltage is superimposed we have $E(t)=E_{0}(1+\epsilon \sin \omega t)$ and $q_{0}=2 \pi^{3} \epsilon_{1} R^{2} E_{0}(1+$ $\left.\epsilon \sin \omega t_{0}\right) / 3$, where $t_{0}$ is the time of the previous impact. The equation of motion of the ball is now, in non-dimensional form,

$$
r \frac{\mathrm{d} \chi}{\mathrm{d} s}=\frac{\left(1+\epsilon \sin \left(\Omega s_{0}\right)\right)(1+\epsilon \sin (\Omega s))}{G} e^{-\left(s-s_{0}\right)}-1,
$$




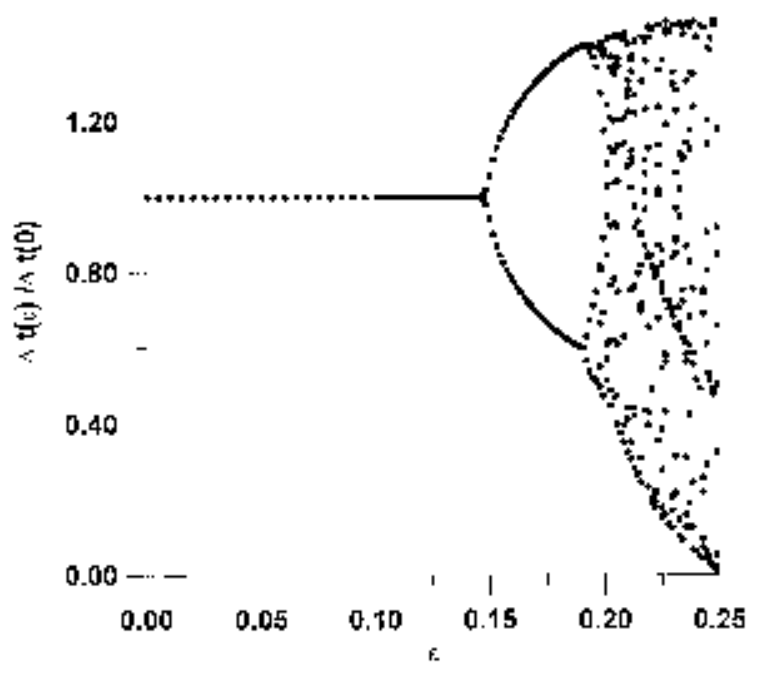

Fig. 3. - Numerical bifurcation diagram for $G=0.56$ and $\Omega=4.8$.

where $s=t / \tau, \chi=z / R$ and $\Omega=\omega \tau$. This equation can be integrated to obtain an implicit equation for the non-linear map:

$$
s_{n}=F\left(s_{n-1}, \epsilon, G, \Omega\right)
$$

for the time $s_{n}$ of the $n$ impact. The bifurcation diagram is obtained iterating the map and representing $s_{n+1}-s_{n}$ as a function of $\epsilon$. In order to avoid the transients, $n$ is taken greater than a certain value, so the obtained values do not depend on the initial $s_{0}$. Figure 3 shows the bifurcation diagram of this non-linear map for $G=0.56$ and $\Omega=4.8$, the frequency associated to the period of the unperturbed motion. The bifurcation occurs at $\epsilon=0.145$. The value of the bifurcation point changes, increasing when $G$ decreases. For instance, the bifurcation occurs at $\epsilon=0.13$ for $G=0.6$ and $\epsilon=0.165$ for $G=0.5$.

An interesting limit of eq. (8) is $G \rightarrow 0$. The electric force at the electrode is so strong that we are in the limit of impulsive motion. The most interesting frequencies are those close to 0 , because $\Delta t_{0} \rightarrow \infty$. In this case the map takes the form

$$
s_{n}=s_{n-1}+\frac{1}{G}+\frac{2 \epsilon}{G} \sin \left(\Omega s_{n-1}\right)+\frac{\epsilon^{2}}{G} \sin ^{2}\left(\Omega s_{n-1}\right) .
$$

For $G \leq 0.01$ the bifurcation diagram obtained with this formula predicts the bifurcation points and branches correctly.

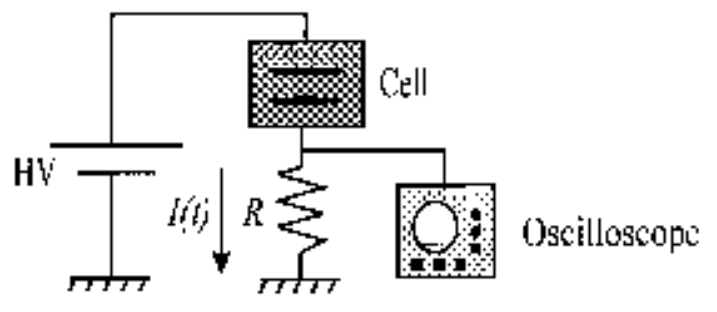

Fig. 4. - Experimental set-up. 

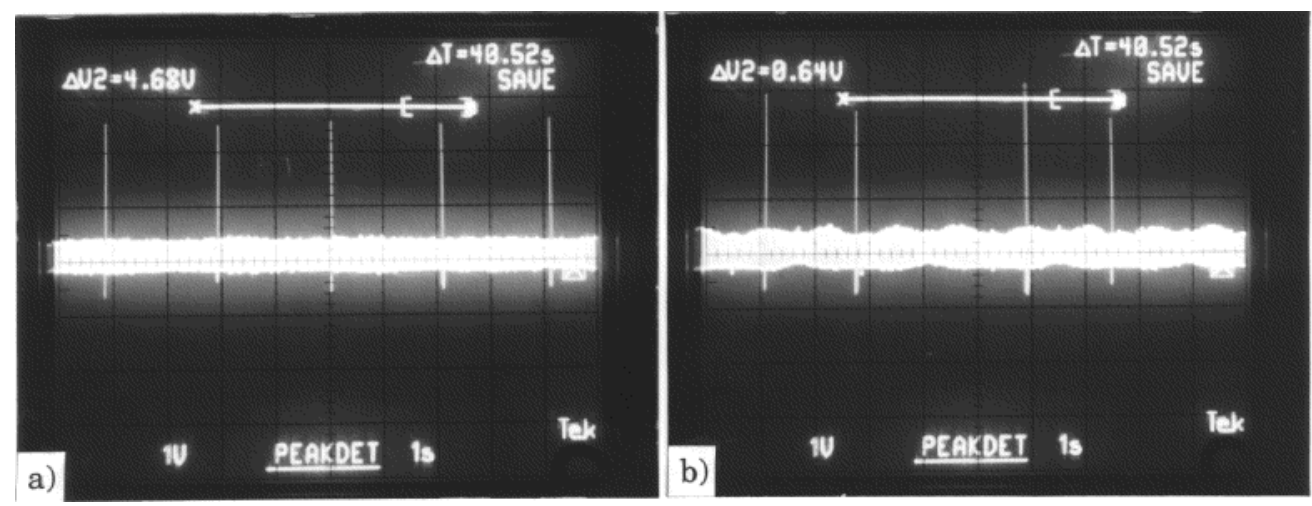

Fig. 5. - Oscilloscope record of the ball impacts. a) Before the bifurcation point. b) After bifurcation.

In the limit $G \rightarrow 0$ our problem resembles other classical ones. The dynamics does not depend on the nature of the driven force, but on the coupling between a bounce and the next one. Similar maps are well documented [1], [2], [6], [7] and have been extensively studied. On varying $\epsilon$ and $\Omega$ a variety of chaotic behavior is expected.

Experiments. - The experimental cell is made of plexiglas and its dimensions are $70 \times$ $70 \times 55 \mathrm{~mm}$. Two parallel aluminum electrodes, circular in shape with diameter $62 \mathrm{~mm}$, are placed inside the cell and the gap between them is $40 \mathrm{~mm}$. A metallic ring, $60 \mathrm{~mm}$ radius and $2 \mathrm{~mm}$ width, rests on the bottom electrode concentric with it, in order to compensate the small divergence of the field at the center of the electrodes. In the absence of this ring the ball drifts sideways at each jump, and finally, after a few jumps, abandons the electrode.

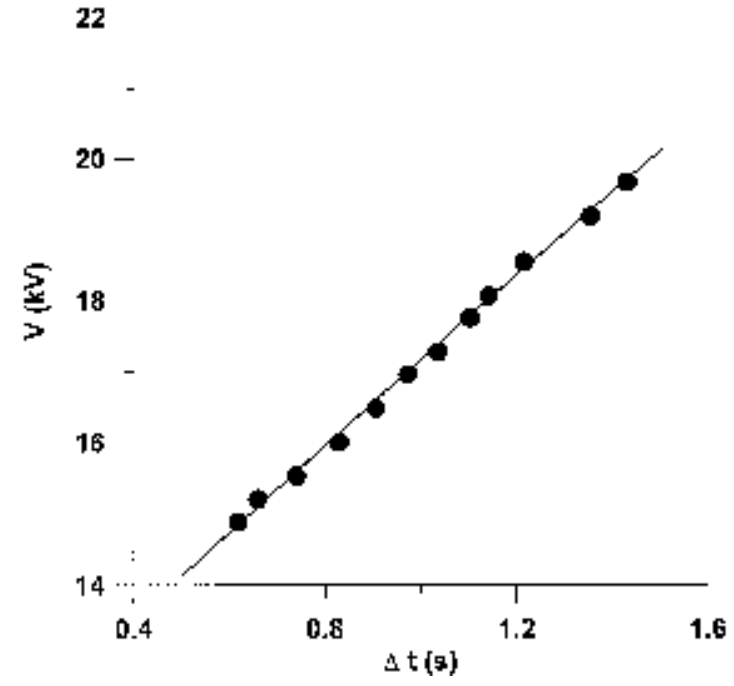

Fig. 6.

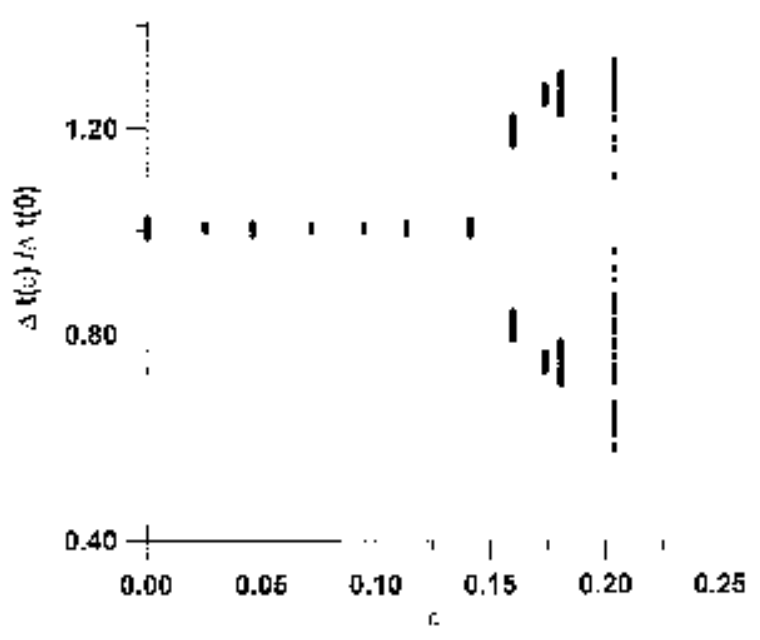

Fig. 7.

Fig. 6. - Applied voltage vs. time between impacts. The solid line is the best fit of the theoretical prediction to the experimental data.

Fig. 7. - Experimental bifurcation diagram. 
The upper electrode is connected to a high-voltage amplifier. The bottom one is connected, either to a storage oscilloscope or, through an amplifier and an acquisition board, to a computer (see fig. 4).

The ball is handmade from aluminum sheet. Its radius is about $3 \mathrm{~mm}$ and its weight about $100 \mathrm{mg}$. The cell is filled up with corn oil, density $990 \mathrm{~kg} / \mathrm{m}^{3}$, viscosity $50 \times 10^{-6}$ $\mathrm{m}^{2} / \mathrm{s}$, conductivity 3 to $5 \times 10^{-11} \mathrm{~S} / \mathrm{m}$ and relative dielectric constant 3.1 . These values imply a relaxation time $\tau=\epsilon_{\mathrm{l}} / \sigma \sim 0.5$ to $0.9 \mathrm{~s}$.

D C voltage. - When a DC voltage is applied, the ball bounces with a very well-defined time of flight. At each impact against the bottom electrode the ball gets charged and a current pulse is registered by the oscilloscope (see fig. 5) or the acquisition board. We storage the time between impacts in the computer for further analysis.

Figure 6 plots the time of flight vs. the applied voltage. The solid line is the best fit to the experimental data of the theoretical equation (6). From this fit we obtain $\tau=0.5 \mathrm{~s}$ and $V_{\mathrm{c}}=11 \mathrm{kV}, V_{\mathrm{c}}$ is the minimum voltage at which the electric force overcomes the gravity and the ball starts to move.

D C plus A C voltage. - When an AC voltage is superimposed, $V(t)=V_{0}(1+\epsilon \sin \omega t)$, all the complexities of non-linear dynamics are present. We have looked for, and focused on, a period-doubling bifurcation. This occurs when the period of the AC voltage coincides with the time of flight of the ball for the applied voltage. If the applied $\mathrm{DC}$ voltage is $V_{0}=17 \mathrm{kV}$, the period is $0.84 \mathrm{~s}$ and the bifurcation takes place at $\epsilon=0.14$. This means that for $\epsilon<0.14$ the ball bounces regularly, with a constant time of flight, whereas for $\epsilon>0.14$ the ball alternates a larger jump with a smaller one. In the diagram $\Delta t v s$. $\epsilon$ this is reflected in the branching at the bifurcation point (see fig. 7). The experimental value of $G$ is $0.6 \pm 0.1$, therefore the numerical map (fig. 3) compares favorably with the experimental one. The bifurcation is clearly demonstrated in the oscilloscope records (fig. 5).

Theoretically, for $G=0.56$ a second period doubling occurs for $\epsilon>0.19$; the experimental accuracy cannot resolve for this second bifurcation but it is almost apparent in the figure.

Conclusion. - A conducting ball in a low-conducting liquid bounces regularly on the bottom electrode when a DC voltage is applied. This system serves as a model for non-linear motion. Adding an $\mathrm{AC}$ voltage of the same frequency as that of the unperturbed system produces a period-doubling bifurcation. This is closely related to the classical problem of a bouncing ball on a moving table.

The authors acknowledge fruitful discussions with Prof. A. CAstellanos and technical assistant from TALLER de la Facultad de Física. This work was carried out with financial support from DGICYT (Spanish Government Agency) PB93-1182.

\section{REFERENCES}

[1] Guckenheimer J. and Holmes P., Nonlinear Oscillations, Dynamical Systems, and Bifurcations of Vector Fields (Springer-Verlag, New York, N.Y.) 1983.

[2] Lichtenberg A. J. and Lieberman M. A., Regular and Stochastic Motion (Springer-Verlag, New York, N.Y.) 1982.

[3] Haus A. H. and Melcher J. R., Electromagnetic Fields and Energy (Prentice-Hall, New Jersey) 1989.

[4] Clift R., Grace J. R. and Weber M. E., Bubbles, Drops and Particles (Academic Press, Harcout) 1978.

[5] FÉLici N. J., Rev. Générale Électr. 75 (1966) 1145.

[6] Kadanoff L. P., Phys. Today, December issue (1983) 46.

[7] Devillard P., J. Phys. I, 4 (1994) 1003. 